\title{
ACERCA DE LAS IDEAS, LA CREATIVIDAD Y LOS PROBLEMAS EN LA PRODUCCIÓN DE CIENCIA'
}

\author{
Orlando Londof̂o Betancourt ${ }^{2}$ \\ Grupo de investigación en Filosofia \\ de la clencia - UAM
}

"El centro del mundo está, definámoslo asi, donde el mundo es pensada. $Y$ el ansia de conocer el mundo està en el miedo por desconocerlo",

Jorge Wagensberg'.

En este escrito me dispongo a demostrar las siguientes tesis:

1. La ciencia no se produce gracias a que existe un bagaje de conocimientos (teorias) y un método para ellas ya establecidos, sino que en muchas ocasiones se logra gracias a lo fortuito, al azar y la creatividad del científico, a la acción súbita del intelecto.

2. No existe un único método para la ciencia, no hay un método para encontrar ideas. No hay un método para el descubrimiento.

3. La ciencia es fundamentalmente encontrar buenas ideas, encontrar problemas y darles solución.
1. texto elaborado en el grupo de investigación en filosofia de ta ciencia uam.

2.profesor, asistente. departamento de fllosofia. universidad de caldas. profesor. asistente. departamento de ciencias humanas. universidad autónoma de manizales. integrante del grupo de investigación en filosofia de la ciencia. uam. trabajo leido en las jornadas filosóficas del departamento de filosofia de la universidad de caldas. banco de la república 26 de septiembre de 2005. y en el foro de filosofía del programa de filosofia de la universidad de caldas, el dia 25 de noviembre de 2005.

3. cf. wagensberg, jorge. ideas sobre la complejidad del mundo. $3^{\circ}$ edición. barcelona. tusquest. 1994, pp 17

\section{A.}

Unversidad Autidnoma de Manizales 
Pensemos en lo que sucede cuando un profesor cuestiona violentamente a un estudiante, por ejemplo a usted, en un curso en que se estudia la evolución de los modelos atómicos, desde los postulados griegos hasta las más contemporáneas teorias, como por ejemplo la de Bohr. ¿Cuál seria la razón para que le llamen la atención?. La razón es que usted insiste en que, a pesar de todas sus bondades, la mecánica cuántica es tan sólo un modelo más de la ciencia; uno como cualquier otro. El profesor del curso se irrita por esta observación que usted ha hecho. A él le parece un irrespeto a su autoridad y al modelo que proclama. Es posible que esto implique que usted decida dejar de una vez por todas la actividad científica y decida dedicarse a otra actividad, por ejemplo, la teología.

Jorge Wagensberg en un hermoso y raro texto publicado en la colección metatemas de Tusquest titulado Ideas para la imaginación impura (53 reflexiones en su propia sustancia) dice que el conocimiento es una representación (necesariamente finita) de un pedazo de la realidad (presuntamente infinita), La ciencia es conocimiento elaborado con el método científico. $Y$ método científico es cualquier método que respete tres principios: el de objetividad, el de inteligiblilidad y el dialéctico. Se es objetivo cuando, ante varias formas de observar un objeto se opta por aquella que menos afecta a la observación. Se es inteligible cuando la representación es, en algún sentido, más compacta que lo representado. $Y$ se es dialéctico cuando el conocimiento se arriesga a ser derribado por la experiencia, El conocimiento es cientifico cuando tienen voluntad de serlo, es decir, cuando logra la máxima objetividad, inteligibilidad y dialéctica... por exiguos que sean tales máximos. Según esto tan científico puede ser un mecánico de carambolas de billar como un 
mecánico cuántico. Según esto, un psicólogo no tiene por qué ser menos científico que un físico... (otra cosa es que renuncie a serlo). De la misma manera, nada hay en contra de que la política, una forma de conocimiento dedicada a organizar la convivencia, se construya con método científico... (otra cosa es que apenas se haya intentado).

La aplicación del método es la parte más previsible, y por lo tanto más planificable, del oficio. Se pueden programar consultas a la naturaleza (experimentos) para descubrir paradojas turbadoras, para medir cómo la realidad se digna encajar en una inteligibilidad o para ensayar diferentes vias de objetividad. Ceder en el método, en honor de cualquier otro beneficio más o menos confesable, es un indicio de fiojera cientifica.

Pero resulta que el método se aplica a una idea. $Y$ no hay un método para cazar ideas, o lo que es lo mismo, todo vale con las ideas: La analogía, el plagio, la inspiración, el secuestro, el contraste, la contradicción, la especulación. El sueño. EI absurdo... Un plan para la adquisición de ideas sólo es bueno si nos tienta continuamente abandonarlo, si nos invita a desviarnos de él, a olfatear a derecha e izquierda, a alejarnos, a girar en redondo, a divagar, a dejarnos llevar por la contingencia... el celebre rigor científico no se refiere a la obtención de ideas sino al tratamiento de éstas. Aferrarse con rigor a un plan de búsqueda de ideas es una anestesia para la intuición.

La ciencia es esencialmente el crecimiento organizado del conocimiento de los hechos. Conforme la ciencia avanza, la información acumulada dia a día se hace cada vez menos 
manejable. Muy pronto no bastarán los tradicionales 3 ó 4 años de formación, sino que los científicos tendrán dedicarle a tal proceso diez o más años, eso si es que quieren capacitarse para estar en el frente de batalla del conocimiento.

Actualmente los cientificos evitan ser aplastados por el enorme peso del conocimiento acumulado, refugiándose en la especialización. El aumento en la especialización es una de las características que distinguen al científico moderno. Debido a ello, los hacedores de ciencia han perdido en forma progresiva la capacidad de comunicarse no sólo con intelectuales de otras disciplinas, sino hasta con los de la propia. Deben esperar que en el futuro ocurra una fragmentación mayor del conocimiento, de modo que cada especialista vivirá en un pequeño mundo propio y personal, que sólo él conoce y que nadie más puede entender. Como diría Toffler, se quiere saber cada vez más sobre cada vez menos cosas.

Pensamientos de este tipo asaltan cuando llega el siguiente ejemplar de la revista especializada y todavía no ha acabado de leer el anterior, o cuando un botánico interesado en la taxonomia entra por equivocación a un seminario de mecánica cuántica. Sin embargo, la opinión resumida arriba es totalmente falsa, en todos y cada uno de sus postulados y premisas. La ciencia no es un catálogo de hechos. Conforme la ciencia avanza, el conocimiento requerido para explicar los hechos disminuye en vez de aumentar, porque las generalizaciones se encargan de incluir cada vez más instancias particulares. Los epistemólogos han señalado que a todas las ciencias les llega la época en que ya no es necesario registrar la caida de cada manzana; basta con formular la ley de la gravitación universal. Respecto a la creciente 
especialización de los científicos, lo opuesto está ocurriendo con la emergencia de ciencias interdisciplinarias, como la biología molecular, la biomecánica entre otras.

Existen otros dos conceptos un poco más sofisticados de la manera como se adquiere el conocimiento científico, que aparentemente son contradictorios. Miremos cada uno de ellos. El primer concepto sostiene que la clencia es una actividad creativa $\mathrm{y}$ esencialmente imaginativa $\mathrm{y}$ que el cientifico participa en una aventura intelectual. Aqui se sostiene que lo más importante es tener buenas ideas, para lo que se necesita gran intulición; realizar los experimentos necesarios para ponerlas a prueba es también importante y requiere mucha precisión, pero lo que tiene prioridad son las ideas, sobre todo las buenas ideas, cuyo origen y mecanismo de generalización nadie conoce. Además, las buenas ideas son producto del individuo más que del equipo, por lo que es indispensable proporcionar al investigador toda la libertad e independencia para que vaya a donde su imaginación lo lleve.

El segundo concepto es el que la ciencia es una actividad esencialmente critica y analitica; el científico es un sujeto que exige el examen más riguroso de los datos antes de emitir una opinión. La imaginación se necesita, pero siempre debe estar sujeta a la censura y al escrutinio objetivo y despersonalizado. La forma óptima de llevar esto a cabo es en equipos o grupos de investigadores, de modo que cualquier tipo de preferencias o sesgos de tipo personal se cancelen. Además, la libertad del investigador no debe confundirse con la terquedad o el libertinaje; si después de algún tiempo no se ha logrado avanzar en la solución de un problema determinado, es bueno que el investigador reoriente sus esfuerzos en búsqueda de 
caminos más productivos.

De acuerdo con el primer concepto, la verdad se genera en la mente del científico, que primero se imagina cómo podrían ser las cosas y después procede a averiguar si realmente son asi. Cada avance científico es el resultado de una aventura especulativa, una excursión a lo desconocido.

Toda investigación parte de alguna cosa, de un esquema conceptual y de un "método". Una idea que ha dado sus frutos consiste en pasar listas a los prejuicios o a las hipótesis de trabajo y romper alguna de ellas, por sólida que pueda ser, a ver que ocurre. Jorge Wagensberg, en un texto introductorio a un coloquio sobre "la imaginación científica", coloca a propósito de lo anterior el siguiente ejemplo:

Se me cayó el anillo en la taza repleta de café y al sacarlo comprobé que estaba totalmente seco.

Puede funcionar el comprobar si el café no estaria en forma de grano dentro de la taza. Los hermanos Wrigth consiguieron por fin volar rompiendo la antigua idea fija de construir ingenios cada vez más estables; su avión fue el primer artefacto diseñado para la inestabilidad (la adecuada, eso si). La consideración de las ideas absurdas da a veces sorpresas, y quizá fuera así como De Broglie concibiera la dualidad onda - corpúsculo. Las ideas que se obtienen por rompimiento brusco de lo establecido conducen por lo general al fracaso, naturalmente, pero cuando triunfan se reconocen como ideas de comprensión súbita (algo muy común en el arte). Es el iajá!, o también liamado ieureka!.

4. WAGENSBERG, Jorge, La clencia esa ficción de la realidad. En: HAKEN, Hermann et al. Sobre la Imaginación clentifica. Traducción de J.M Llosa y otros. Barcelona. Tusquets. 1990. Pp 11 
Recordemos "la navaja de Occam": este filósofo del siglo XIV expuso la idea de que son preferibles aquellas teorias que contienen el menor número posible de presupuestos arbitrarios.

En cambio, en el segundo concepto la verdad reside en la naturaleza y sólo se conoce por medio de las experiencias de nuestros sentidos; la labor del científico es la exploración de la realidad y es a partir de los datos objetivos que se generan niveles de comprensión más generales, o sea el conocimiento.

La antítesis entre estos dos conceptos puede resumirse diciendo que, en el primer concepto, el investigador inventa la realidad, mientras que para el segundo concepto, el científico la descubre.

Estas dos formas de adquísición del conocimiento científico no son opuestas sino complementarias; ambas ocurren durante la investigación, unas veces en forma sucesiva y alternada, otras de manera casi simultánea. Cada uno de ellos se aplica a una parte distinta del proceso cientifico, que consiste en tener ideas y ponerlas a prueba. Ése es el llamado "método científico": tener ideas $y$ ponerlas a prueba. Encontrar problemas y resolverlos, como diria Popper:

...No hay más que un camino hacia la ciencia o hacia la filosofia, en este sentido, encontrar un problema, ver su belleza y enamorarse de él; casarse con él y vivir feliz con él, hasta que la muerte os separe, al menos que uno encuentre otro problema más fascinante aún, o a menos, naturalmente, que encuentre una solución. Pero incluso si uno encuentra solución, puede descubrir entonces, para 
su delicia, la existencia de toda una familia de encantadores, aunque quizá dificiles, problemas, hifos por cuyo bienestar puede trabajar con un objetivo, hasta el fin de sus dias."

Y no olvidemos que Popper fue, tal como el lo dice en el "objetivo y el realismo de la ciencia", el único profesor durante más de treinta años, de una disciplina inexistente, la "metodología de la investigación".

Tener ideas es un acto imaginativo, que ocurre en la mente individual, por mecanismos no bien conocidos y muy de tarde en tarde. Cuando se le preguntó a Malenbrot cómo hacía para tener buenas ideas, respondió con dos fórmulas, en lugar de una: la primera fue, tener muchas ideas y eliminar las malas, de modo que al final se queda uno con las buenas; la segunda fórmula fue todavia mejor, dijo: "preguntarle a mi esposa, ella siempre tiene puras buenas ideas". No olvidemos que Kekule concibió fantaseando mientras dormitaba en un autobús, la estructura hexagonal del benceno. En una entrevista a Einstein, un reportero le preguntó:

¿Puede usted decirme cómo hace investigación?". A lo que Einstein contestó: "Bien, mire usted, me levanto temprano y me voy a caminar al bosque...". "iAh!, dijo el entrevistador, usted encuentra la inspiración en la naturaleza..." "No precisamente, contestó Einstein, sino que me gusta caminar entre los árboles..." "Bueno, dijo el reportero, y llevará usted una libreta para anotar las ideas que se le vayan ocurriendo...?. "No, contestó Einstein, riéndose, se me ocurren tan pocas que valgan la pena, que no necesito apuntarlas para acordarme de ellas..."

5. POPPER, Karl. Realismo y el objetivo de la clencia. Traducción de W.W. Bartley III. Madrid. Tecnos. 1985. Pp 48. 
Por otro lado, poner las ideas a prueba es lo que llamamos predicción o experimento, y estaremos de acuerdo en que hay que hacerlo con la mayor rigurosidad y cuidado posibles y tantas veces como sea necesario para convencernos de que posiblemente asi sean las cosas. Creo que así es como se adquiere el conocimiento científico.

Edgar Morin, escribe sobre concepto de imaginación en la ciencia la siguiente manera:

La imaginación, la fluminación, la creación, sin las cuales el progreso de la ciencia no hubiera sido posible, no entraban en las ciencias más que ocasionalmente: eran, lógicamente, no dignas de atención,y, epistemológicamente siempre condenables. Se ha hablado de ellas en las biografias de los grandes sabios, pero nunca en los manuales y los tratados, en los que por lo tanto, una sombría compilación estaba constituida, como en los yacimientos subterráneos de carbón, por la fosilización y la comprensión de aquellos que, en su origen, habian sido fantasias, hipótesis, proliferación de ideas, inventos, descubrimientos. ${ }^{\text {? }}$

Extendámonos un poco más sobre estas dos caras del supuesto "método científico", la generación de las ideas y su confrontación con la realidad. Me valdré para el caso, de la analogía entre el tema en discusión y dos conocidos mitos de la literatura clásica."

Sísifo, como ustedes recuerdan, era el hijo del dios Eolo y de la ninfa Enerate, y a su vez el padre del dios del mar; Glauco, era rey de Corinto y siempre se le

6. HAKEN, Hermann et a l. Sobre ta I $m$ a $g$ I $n$ a clón clentifica. Op cit.

7. MORIN, Edgar. Introducción al pensamiento complejo. Traducción de Marcelo Pakman. Barcelona. Gedisa, 1996. Pp 83.

8. CF Diaz Ruy, La lechuza de Minerva. México. F.C.E. 1999. 
representa como un joven atlético, en plena juventud y fuerza. Cuando la muerte vino por él, Sisifo la encadenó de modo que nadie moriria hasta que Ares la liberó y le entregó al rey. Cuando Sísifo llegó al país de los muertos fue castigado en forma peculiar; debía empujar una piedra enorme hacia arriba, por la ladera de una colina alta y empinada, y cuando ya estaba casi por llegar a la punta, la pledra se le escapaba y rodaba hasta el valle, por lo que Sisifo debia bajar y volver a empujarla por toda la eternidad. Este mito adquirió gran popularidad en los 60's gracias a un pequeño ensayo que Albert Camus escribió sobre él, caracterizándolo como héroe absurdo, condenado a trabajar toda la eternidad sin lograr nunca nada, en castigo por su pasión por la vida.

En este mito, uno percibe el esfuerzo total del cuerpo, empujando para elevar la enorme pledra, rodarla y subirla por la pendiente cientos de veces: uno ve la cara contraida, la mejilla apoyada contra la piedra, el hombre cargando la masa cublerta de lodo, el ple haciendo palanca, al principio con los brazos extendidos, la potencia concentrada en las manos llenas de tierra. Al final de su prolongado esfuerzo, medido en un espacio sin cielo y en un tiempo $\sin$ fondo, el objetivo casi se cumple. Pero en ese momento Sísifo ve la piedra rodar hacia abajo en unos cuantos momentos, hacia las profundidades desde donde tendrá que empujarla otra vez hacia la cumbre. Lentamente, Sísifo baja también hacia el valle.

La tragedia de Sisifo no es que su trabajo sea estéril y que no alcance nunca nada; lo trágico es que lo sabe, que está consciente de lo vacio de sus esfuerzos, de la inutilidad que se extiende frente a él como un mar 
infinito, por toda la eternidad. No importa cuántos siglos pasen, hasta el mismo fin de todos los tiempos, Sísifo estará empujando su roca cuesta arriba, sabedor de que nunca llegará a la cima de la colina. Al menos que el acto mismo de empujar la piedra se transforme en un fin, seguirá careciendo para siempre de sentido. No hay nada en la repetición ad infinitum que se lo confiera.

En cambio, la historia de Penélope es diferente. Como ustedes recuerdan, en la Odisea de Homero Penélope es la esposa de Ulises. Se trata de una mujer noble, hija de fcaro de Esparta y de la ninfa Peribea. Después de la caida de Troya, y durante la larga ausencia de su esposo, varios nobles de Itaca y de las islas vecinas la asediaban para que se casara con uno de ellos. Penélope los mantiene a distancia con la promesa de que haria su elección al terminar de tejer una manta para Laertes, el viejo padre de Ulises. Durante el día, Penélope tejía con gran destreza y primor, frecuentemente bajo el acecho de sus pretendientes, pero ai amparo de la noche destejia todo lo que habia adelantado, para volver a empezar al dia siguiente. El truco le duró tres años, hasta que sus sirvientes revelaron el secreto a los pretendientes.

La llegada de Ulises, disfrazado de viejo y solamente reconocido por su perro, es uno de los primeros "Finales Felices" de la historia. Penélope nos interesa porque a primera vista le pasa lo mismo que a Sisifo: lo que teje durante el día lo deshace en la noche, para volver a empezar el mismo ciclo al dia siguiente.

También como Sísifo, Penélope está consciente de su problema, que se extiende frente a ella por un tiempo indeterminado: ella ignora cuándo regresará Ulises. 
Sin embargo, hay dos grandes diferencias entre Sisifo y Penélope: la primera es que ella realiza su trabajo con otra meta en mente, el destejido nocturno tiene el objetivo de no terminar de tejer la manta, pero esto no es un fin en sí mismo sino que es simplemente un medio para alcanzar su verdadero fin, que es mantener a raya a sus pretendientes mientras Ulises regresa. La segunda diferencia es que Penélope triunfa todos los dias, su trabajo se ve coronado por el éxito, cada noche regresa sola a su lecho, a destejer la manta y a esperar a Ulises. En cambio, Sisifo no tiene un objetivo ulterior en su terrible trabajo, ni la posibilidad de alcanzar alguna vez el éxito, suponiendo que lograr subir la roca hasta la cima fuera lo suficientemente atractivo para justificar una eternidad de esfuerzos. Podemos imaginarnos a Penélope satisfecha con el éxito de su estrategia, mientras que Sisifo maldice la inutilidad de sus esfuerzos.

Estas dos diferencias se basan en que Penélope trabaja en función de una idea, tiene un objetivo bien definido, basado en una serie de suposiciones. Las suposiciones son casi perfectas; el casi es porque se equivocó en la fidelidad de sus esclavos, pero el error no tuvo mayores consecuencias gracias al fino sentido teatral de Homero. En cambio, Sísifo no tiene ninguna idea, ningún objetivo ulterior que desee alcanzar por medio de su trabajo, que es empujar la maldita piedra. Cuando alguien, en algún laboratorio, dice: "vamos a hacer esto a ver qué pasa...", Sisifo se siente aludido, se satisface de ser imitado, se regocija de estar ganando reclutas para su forma estéril de trabajar, de empujar una piedra sin ninguna idea en la cabeza. La figura de Sișifo puede ser trágica o épica, pero de ninguna manera poética, porque la poesia requiere la participación de

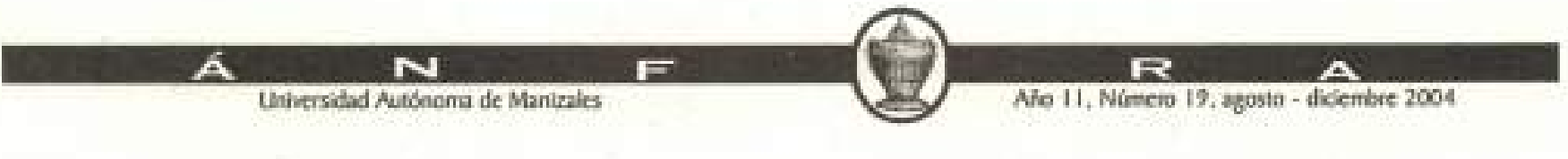


las emociones y del intelecto, y de todo esto el pobre Sísifo no sabe nada.

Asi pues, no queda otra instancia que advertir que el proceso de conocimiento es, al mismo tiempo, condicionado e incondicionado, histórico y lógico, diacrónico y sincrónico. Dicho de otro modo: es histórica y socialmente condicionado cuando y bajo algunas circunstancias una sociedad, un individuo o un conjunto de individuos establecen una ley, elaboran un método, realizan un descubrimiento o construyen una teoría científica, pero es incondicional la validez de sus tesis.

Podemos datar las condiciones bajo las cuales surge la geometría euclidiana, pero la validez relativa de la geometria de Euclides no puede ser negada, dentro de las necesarias normas de sus límites conceptuales. Esto es que un conocimiento verdadero posee un carácter al mismo tiempo absoluto y relativo. Por ejemplo, hay quienes en la actualidad para negar la posibilidad de un conocimiento verdadero, se preguntan, por decir algo, qué es el "norte" y negar que haya algún "norte" absoluto, puesto que el norte de unos es el sur para otros. Ambas formulaciones son correctas porque una es el limite de la otra. Son verdaderas precisamente en su relatividad (en su relación). Establecer esas coordenadas no destruye el carácter absoluto de la proposición, sino que lo relativiza.

Podemos en consecuencia, mostrar cómo y por qué ciertas teorias y ciertos métodos surgieron en ciertas sociedades, es decir, qué condiciones, históricas y sociales las hicieron posibles. Pero, si hacemos esto, nos limitamos a los enunciados 
de una sociología (de la ciencia, del arte o de la filosofía). Lo más importante queda aún por ser realizado, a saber, la determinación de cómo y por qué mantienen su vigencia. En este sentido, quiero destacar que lo más importante de todo conocimiento reside en ampliar el campo de nuestra ignorancia. Saber que no sabemos constituye siempre el primer paso hacia el conocimiento.

En este sentido se podrian interpretar las siguientes palabras del profesor Mexicano Ruy Díaz Tamayo cuando dice:

La clencia comparte con la política, la industria, la ingeniería, el metro y el servicio telefónico, una obligación fundamental: la de producir resultados concretos y objetivos, la de funcionar. Al margen de su inmenso valor cultural y de su enorme contribución al avance de la civilización, el trabajo científico de Pasteur también sirvió para establecer un método general de preparación de vacunas, por medio de gérmenes de virulencia experimentalmente atenuada. Este método ha funcionado muy bien, ya que siguiendo la idea de Pasteur se han producido vacunas eficientes para varias enfermedades infecciosas, $y$ los resultados benéficos obtenidos no pueden considerarse como una "construcción social", en vista de que las vacunas tienen el mismo efecto en sociedades tan distintas como los grupos gay de New York y los indigenas zapotecas de la Sierra de Oaxaca. En otras palabras, el conocimiento que surge de la ciencia no está determinado, como postulan Latour y 
Woolgar, nada más socialmente; su contenido no depende en exclusiva de la estructura y el estilo de la sociedad en la que se desarrolla. Desde tiempo inmemorial, la ciencia también ha dependido, no sólo para definir sus áreas de trabajo sino para enjuiciar sus resultados, de su contacto con la realidad..."

Hoy, por ejemplo, un muchacho que haya pasado por la escuela secundaria "sabe" más de geometria y de matemáticas que Euclides, Descartes o Leibniz. Pero ese "saber" es un saber recibido. $Y$ a veces esos conocimientos no son científicos, $y$ ellos mismos no lo son, como si lo fueron Pitágoras, Kepler o Newton. La ciencia avanza desde lo que se sabe hacia lo que no se sabe 0 , a la inversa, desde la ignorancia hacia el conocimiento. Poner en duda es el signo inequivoco de la auténtica actitud científica; su rasgo más acusado: la crítica, la investigación, el hallazgo y la innovación. La ciencia, por ello mismo, puede no ser enseñada (como no pueden ser enseñados el arte o la filosofía). Lo que se enseña en las escuelas es, en el mejor de los casos, una técnica, una historia que, una vez dominadas, se convierten en un instrumento para los verdaderos quehaceres científicos (o artísticos o filosóficos). Ciencia y técnica se oponen en este sentido profundo. Nadie recibe titulo de "poeta" en una escuela de letras; nadie tampoco el de "ontológo" en una escuela de filosofía, ni el de "científico" en una escuela de física. Estos títulos se conquistan y son, en ocasiones, el fruto de toda una vida.

En este sentido el conocimiento como la apropiación estructural del mundo debe permitir que el mundo sea

9 TAMAYO PEREZ, RUY. Op cit. Pp 274275. 
transformado, es decir, resignificado.

El conflicto de creer profundamente en una verdad con la comprobación de que ésta es insostenible, es de hecho el conflicto entre el deseo de crear y lo que podriamos llamar la honestidad intelectual. Es decir, la capacidad que frente al conocimiento debemos de tener, como diria Popper, de aceptar que estoy en un error y que tú tienes la razón. Que la verdad está determinada por quien coloque sobre la mesa los mejores argumentos, y en sostener discusiones racionales de la manera más impersonal posible. Es decir, hay que mantener un espiritu de diálogo libre. Se necesita que cada persona sea capaz de mantener varios puntos de vista, a manera de suspensión activa, y al mismo tiempo tratar las ideas de los otros con el cuidado y la atención que presta las propias. No se exige a cada persona que acepte o rechace puntos de vista determinados, sino que más bien debe intentar comprender lo que significan.

De lo anterior no queda sino citar, para terminar, un texto de Karl Popper en el que se se muestra la esencia de lo que aquí hemos querido plantear:

Yo soy un racionalista. Por racionalista entiendo un hombre que quiere comprender el mundo y aprender mediante la discusión con otros... Por "discutir con otros" entiendo, más concretamente, criticarlos, suscitar sus criticas y tratar de aprender de ellas. EI arte de la discusión es una forma peculiar del arte de la lucha, con palabras en vez de espadas, e inspirado por el interés de acercarse a la verdad sobre el mundo. 
No creo en la actual teoria de que para que una discusión sea fructífera, los discuten tengan que tener mucho en común. Por el contrario, creo que cuánto más distinta sea su formación, más fructífera será la discusión. Ni siquiera hace falta empezar por tener una lengua común: si no hubiera existido la Torre de Babel hubléramos tenido que construirla. La diversidad hace más fructífera la discusión crítica. Las únicas cosas que deben de tener en común los participantes en una discusión son el deseo de saber y la disposición a aprender del otro, mediante la critica severa de sus concepciones - en la versión más fuerte que sea posible dar a sus concepciones y escuchando to que él tienen que decir como replica.

Creo que el llamado método de la ciencia - continúa diciendo Popper - consiste en este tipo de crítica. Las teorias clentificas se distinguen de los mitos simplemente en que pueden criticarse y en que están abiertas a modificación a la luz de las críticas. No pueden ni verificarse ni probabilificarse.

Mi actitud critica - 0 , si ustedes prefieren, herética Influye, naturaimente, en mi actitud respecto a mis colegas filósofos.

Todos conocerán la historia del soldado que descubrió que nadie en su batallón (menos él, claro) llevaba el paso. Yo mismo me encuentro constantemente en esta divertida posición. $Y$ tengo mucha suerte porque, por lo general, algunos de lo otros miembros del batallón están bastante bien dispuestos a llevar el paso. Esto aumenta la confusión: y como no soy un admirador de la disciplina en filosofia, estoy satisfecho mientras haya bastantes miembros del 
batallón que hayan perdido el paso suficientemente los unos respecto a los otros. ${ }^{10}$

\section{BIBLIOGRAFÍA.}

HAKEN, Hermann et al. Sobre la imaginación científica. Traducción de J.M Llosa y otros. Barcelona: TusQuets. 1990.

POPPER, Karl. Realismo y el objetivo de la clencia. Traducción de W.W. Bartley III. Madrid: Tecnos. 1985.

MORIN, Edgar. Introducción al pensamiento complejo. Traducción de Marcelo Pakman. Barcelona: Gedisa, 1996.

TAMAYO PEREZ, RUy. ¿Existe el método científicoi?. (Historia y realidad). México: F.C.E. 1990.

WAGENSBERG, Jorge. Ideas sobre la complejidad del mundo. $3^{\circ}$ edición. Barcelona: Tusquest. 1994.

. Ideas para imaginación impura (53 reflexiones en su propia sustancia). Barcelona: TusQuets. Metatemas. 1998.

BOHN, David y PEAT David. Clencia, Orden y Creatividad (las raices creativas de la ciencia y la vida)Traducción de Joseph M. Apfelbäume. Barcelona: Kairos. 1988.

10. POPPER, Karl. EI Realismo y el Objetivo de la ciencia. Op cit. Pp $46-47$ 